\title{
Humanización un desafío en instituciones públicas del departamento de Córdoba, Colombia
}

\section{Humanization in health care: a challenge in health Public Institutions in Córdoba, Colombia}

\author{
Martha Orozco Valeta ${ }^{1}$ (D) Martha Medina Payares ${ }^{2}$ Martha Montoya Vega ${ }^{3}$ \\ Recibido para publicación: Febrero 7 de 2019 - Aprobado para publicación: Junio 8 de 2019
}

\begin{abstract}
RESUMEN
Objetivo. Evaluar la atención humanizada en instituciones prestadoras de servicios de salud públicas de cinco (5) municipios del departamento de Córdoba (Montería, Sahagún, Lorica, Planeta Rica y Montelíbano) en el año 2016. Materiales y métodos. Investigación descriptiva, transversal de tipo mixto; para el desarrollo de este estudio se emplearon dos técnicas de recolección de datos; primero la aplicación del instrumento creado por Willmer Emilio Mojica Gutiérrez en su tesis de grado: "La Gerencia social en la humanización de la atención en salud" el cual es gratuito y de libre acceso. Como segunda herramienta la observación. Resultados. El mayor porcentaje de cumplimiento se obtuvo para la variable trato humanizado a discapacitados y enfermos mentales (81\%); seguida de servicios generales (68\%); destacando el $100 \%$ en el cumplimiento de la variable seguimiento en todos los prestadores evaluados. Conclusión. La humanización debe ser un compromiso institucional liderado desde la alta gerencia, que promueva la transformación cultural en los colaboradores; además requiere de la asignación de recursos financieros para la adecuación de infraestructura, la inclusión de elementos de humanización en el ambiente físico, la planificación de cronogramas de capacitación y aplicación de estrategias de sensibilización que garanticen el fomento del trato humanizado.
\end{abstract}

Palabras clave: Equidad, atención en salud, humanización, acreditación en salud

\begin{abstract}
Objective. To evaluate the humanized care service in public health providers in five (5) municipalities of the department of Córdoba (Montería, Sahagún, Lorica, Planeta Rica and Montelíbano) in 2016. Materials and methods. Descriptive, cross-sectional mixed type research; to carry out this study, two data collection techniques were used; first the application of the instrument created by Willmer Emilio Mojica Gutiérrez in his thesis: "Social management in the humanization of health care" which is for free and can be downloaded, and the observation. Results. The highest percentage of compliance was obtained to the humanized treatment variable for disabled and mentally ill people ( $81 \%)$; followed by general services attention (68\%); highlighting $100 \%$ of compliance with the follow-up variable in all health providers evaluated. Conclusion. Humanization must be an institutional commitment led by high management chiefs, who should promote cultural transformation in employees; it also requires the allocation of financial resources for the adaptation of adequate infrastructure, the inclusion of humanization elements in physical environments, in planning schedules of training and application of awareness strategies that guarantee the promotion of a more human treatment.
\end{abstract}

Keywords: Equity, health care, humanization, health accreditation.

\section{Citación (Vancouver)}

Valeta OM, Madina PM, Montoya VM. Humanización un desafío en instituciones públicas del departamento de Córdoba, Colombia. Rev Avances en Salud; 2019. (3)1:1625. DOI: $10.21897 / 25394622.1749$ (c) 2019. Universidad de Cordoba. Este es un artículo de acceso abierto distribuido bajo los términos de la licencia Creative Commons Attribution License, que permite el uso ilimitado, distribución y reproducción en cualquier medio, siempre que el autor original y la fuente se acreditan.

\footnotetext{
1 Esp. en Enfermería Médico Quirúrgica, Facultad Ciencias de la Salud. Universidad de Córdoba, Montería, Colombia

2 M.Sc. en Gestión de la prevención de riesgos laborales,la excelencia, el medio ambiente y la responsabilidad corporativa, Facultad Ciencias de la Salud, Universidad de Córdoba, Montería, Colombia.

${ }^{3}$ M.Sc. en Salud Pública, Facultad Ciencias de la Salud. Universidad de Córdoba, Montería, Colombia. Correspondencia: marthamontoya@ correo.unicordoba.edu.co
} 


\section{INTRODUCCIÓN}

El diseño de herramientas que ayudan al hombre a realizar mejor sus actividades a través de la tecnología, se ha convertido en soporte vital de la sociedad, en el sector salud no puede ignorarse que el desarrollo tecnológico brinda y garantiza al paciente procedimientos más seguros; sin embargo, también ha contribuido a que los valores implícitos en el trato entre seres humanos se estén dejando de lado (1).

Los grandes avances científicos y tecnológicos no solamente traen como consecuencia un considerable aumento de la esperanza de vida, sino también una forma de tratar al paciente que puede llegar a producir una especie de "cosificación" de la persona que se aleja de una visión de conjunto de la misma, que repercute en una deshumanización de la asistencia sanitaria (2).

Cuando se habla de humanizar la atención en salud, se hace referencia en principio, a todas aquellas acciones que hacen más humano al hombre, y por ello las instituciones hospitalarias como subsistemas sociales, cumplen la función de socializar a los individuos con pautas, normas establecidas, adaptándolos e integrándolos al sistema, de esta forma el cuidado holístico se ve dificultado por las múltiples tareas delegadas de tipo biomédico, quedando relegadas acciones como la comunicación eficaz y el interactuar con el paciente y su familia (3).

En la humanización de la atención en salud es fundamental que todos los profesionales orienten su conocimiento al servicio de los demás, situando en primer lugar la sensibilidad y la ética en el acto del cuidado, resaltando la dignidad humana y ofreciendo una óptima atención, comunicación e información a los usuarios, pacientes, familias y otros profesionales involucrados en el sistema (4).
Es por esto, que las instituciones prestadoras de servicios de salud (IPS), por su carácter misional, no deben olvidar la esencia de su atención, la cual debe estar inmersa en una asistencia más humana, con respeto y una actitud de acogida al paciente; enfocada en la verdadera razón de ser de estas organizaciones, el ser humano; entendiendo, que el objetivo de la atención en salud, no solo es brindar conocimientos teóricos y prácticos al paciente y su familia, sino garantizar el desarrollo de competencias de sus colaboradores para alcanzar un crecimiento humano y profesional que asegure la prestación de un servicio con los mayores estándares de calidad, donde la atención esté centrada en el trato humano para los pacientes (5).

Para el Ministerio de Salud y Protección Social de Colombia, "La humanización es concebida como un imperativo ético para el Sistema de Salud, y no solamente como un atributo del servicio, producto o información, de cada una de las organizaciones o actores que lo conforman" (6).

En Colombia, existe un marco normativo sustancioso en lo concerniente a la humanización del servicio, la ley insta a IPS para que en su proceso de planeación de la atención, contemplen el diseño de una política de atención humanizada que incluya criterios para el paciente y el personal de la institución, (7) tales como el Decreto 1011 de 2006 (8) en su componente de habilitación con la Resolución 2003 de 2014 (9) que exige a los prestadores de servicios de salud, contar con un programa de seguridad del paciente, que genere una atención en salud segura, esto con la intencionalidad de la prestación de un servicio digno y con respeto como primer eslabón en la humanización de la atención, y en su componente de acreditación que busca con la implementación del Sistema Único de Acreditación (SUA) a través de la Resolución 123 de 2012 (10) una atención centrada en la persona, desplegando la humanización como eje transversal a todo el sistema 
obligatorio de garantía de la calidad en salud (SOGCS).

Es de aclarar que la implementación del SUA no es de obligatorio cumplimiento para los prestadores de salud, lo obligatorio específicamente para la IPS públicas es la implementación de la ruta crítica contemplada en el plan de mejoramiento de la calidad en salud (PAMEC) como punto de partida para la acreditación en salud (11).

Basados en lo anterior, y en la importancia de la prestación de servicios de salud de excelente calidad con atención centrada en la persona; y ante la inexistencia de estudios que evalúen la humanización bajo un enfoque integral, surgió la necesidad de realizar este estudio para evaluar la atención humanizada en IPS públicas de cinco (5) municipios del departamento de Córdoba (Montería, Sahagún, Lorica, Planeta Rica y Montelíbano) en el año 2016 y de acuerdo a los resultados obtenidos diseñar estrategias para el fortalecimiento de la cultura del trato humanizado en las IPS objeto de estudio.

\section{MATERIALES Y MÉTODOS}

Tipo de estudio. Se realizó una investigación descriptiva, transversal de tipo mixto, en ocho IPS públicas de cinco municipios del departamento de Córdoba en el año 2016. El criterio de selección utilizado para la escogencia de los municipios hace referencia al área de influencia de estos. (Figura 1).

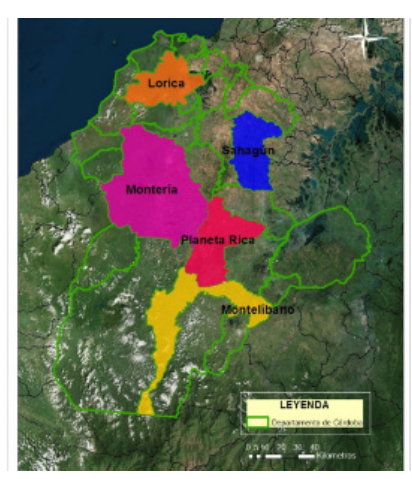

Figura 1. Departamento de Córdoba, área de influencia por municipios. Fuente: Elaboración propia.

Con la finalidad de cumplir con los principios de privacidad y confidencialidad en las diferentes instituciones involucradas, se asignó a cada una un código de identificación como se observa en la (Tabla 1).

\begin{tabular}{lc}
\hline \multicolumn{1}{c}{ Municipio } & Código \\
\hline Montería & 1 \\
Montería & 2 \\
Sahagún & 3 \\
Sahagun & 4 \\
Lorica & 5 \\
Lorica & 6 \\
Montelíbano & 7 \\
Planeta Rica & 8 \\
\hline
\end{tabular}

Tabla 1. Codificación de las IPS evaluadas. Fuente: elaboración propia.

Obtención y análisis de la información. Para la recolección de la información como fuentes de información secundarias, se utilizó toda la normativa asociada con el trato humanizado, específicamente la Resolución 123 de 2012, que en su artículo segundo adopta los manuales de acreditación, tales como el manual de acreditación en salud ambulatorio y hospitalario donde reposan los estándares relacionados con el tema.

Además, se hizo necesario observar el consolidado de indicadores que se reportan a la plataforma de intercambio de información (PISIS) del sistema integral de información de la protección social (SISPRO), por ser la herramienta que permite monitorear la calidad de atención en salud, así como también documentos propios de las IPS objeto de estudio, como su portafolio de servicios y certificado de habilitación entre otros. 
Como fuentes primarias, se emplearon dos técnicas de recolección de datos: una lista de chequeo para evaluar de forma integral el trato humanizado en la atención en salud, instrumento creado por Willmer Emilio Mojica Gutiérrez (12) en su tesis de grado: "La Gerencia social en la humanización de la atención en salud" que reposa en la Biblioteca Virtual de la Escuela Superior de Administración Pública el cual es gratuito y de libre acceso; dividida en tres secciones:

Sección 1: variables según condiciones y situación del paciente (grupos etarios, binomio madre/hijo, discapacitados y enfermos mentales, enfermos terminales, fallecimiento y abandono).

Sección 2: variables relacionadas con los funcionarios (profesionales de los servicios de la salud, personal de vigilancia y servicios generales).

Sección 3: variables relacionadas con la prestación del servicio (atención en los servicios de urgencias, ingreso y egreso de usuarios, prestación de la atención y seguimiento).

La aplicación del instrumento fue dirigida a diferentes profesionales, técnicos y/o auxiliares en salud, así como también a personal de servicios generales y de vigilancia de acuerdo a la sección evaluada, como constante se tuvo la presencia del coordinador o líder de calidad en cada una de las IPS evaluadas. Para evitar la subjetividad y/o presunción de hechos en las respuestas obtenidas, se utilizó como segunda herramienta la observación, que permitió evidenciar la realidad directamente sin intermediación percibiendo la situación estudiada tal como es.

Es de resaltar, que inicialmente se efectuó una visita para la presentación del proyecto de investigación a realizar con el fin de sensibilizar al representante legal o a su delegado sobre la importancia de este y su objetivo, así mismo se identificaron los profesionales a entrevistar para la aplicación de la lista de chequeo como el coordinador o líder de Calidad, el coordinador médico y jefe de enfermería, entre otros. Aspectos éticos. Según la resolución 08430 de 1993 la investigación se clasifica como un estudio sin riesgo, así como también cuenta con el consentimiento por escrito de cada institución participante del estudio y de los funcionarios participantes.

\section{RESULTADOS}

El análisis de datos se realizó mediante estadística descriptiva arrojando proporciones y valores porcentuales para cada una de las variables contenidas en las secciones del instrumento a aplicar, después se procedió a evaluar los resultados obtenidos en cada IPS comparándolos con la totalidad de la población objeto de estudio, para finalmente diseñar un plan de mejoramiento ajustado a las necesidades identificadas en cada escenario intervenido con miras al fortalecimiento de la atención humanizada en las IPS objeto de estudio.

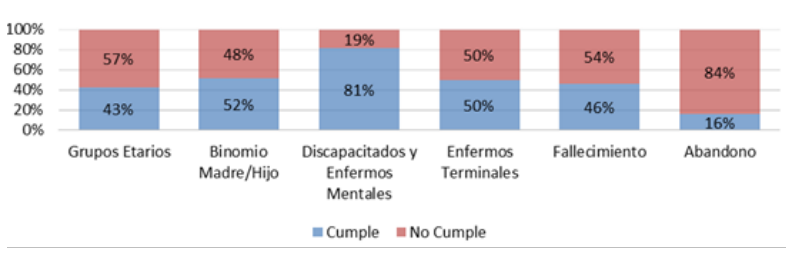

Gráfica 1. Porcentaje de cumplimiento del trato humanizado por las condiciones y situación del paciente.

Fuente: Información de las IPS objeto de estudio.

Sección 1: Los resultados indican el mayor porcentaje de cumplimiento para el trato humanizado a discapacitados y enfermos mentales con un $81 \%$, mientras que la variable abandono arroja el menor porcentaje equivalente a un $16 \%$, sin embargo, cabe anotar que el cumplimiento de las variables restantes oscila en un $50 \%$, valor que evidencia la necesidad inmediata de intervención para mejorar la atención 
» Diseñar estrategias lúdicas para la atención de la población infantil.

» Realizar evaluación de expectativas y necesidades del paciente en relación con su patología y tratamiento

» Brindar consejería y apoyo emocional al paciente y la familia, cuando no hay respuesta al tratamiento, realizar

Grupos etarios la preparación a todo el grupo familiar para la muerte.

» Definir criterios para brindar apoyo emocional a la familia cuando se presenta muerte cerebral, retiro de soporte vital, decisiones de no tratamiento y no reanimación.

» Educar al padre que entrará al parto en las medidas de

Binomio madre/hijo

Discapacitados y enfermos mentales bioseguridad que debe guardar.

» Contar con personal de salud idóneo para brindar una atención cálida y afectuosa.

" Contar con un protocolo para realizar abordaje integral al paciente con dolor.

Enfermos terminales

» Participación activa de los pacientes en la toma de decisiones sobre los planes terapéuticos del cuidado paliativo.

Fallecimiento

Abandono
» Establecer la inclusión de la familia en un programa de duelo.

" Conformar un programa de redes de apoyo, con participación activa de la comunidad.

" Diseñar un protocolo de búsqueda de la familia del usuario, el cual puede ser contemplado dentro del programa de redes de apoyo.

Tabla 2. Plan de mejora. Fuente: elaboración propia.

Con relación a la segunda sección, en la gráfica 2 se observa el porcentaje del cumplimiento del trato humanizado por parte de los funcionarios.

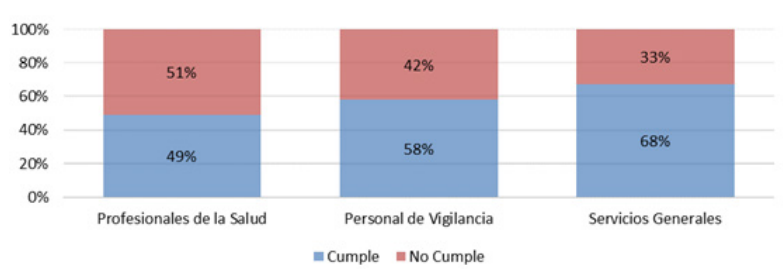

Gráfica 2. Porcentaje de cumplimiento del trato humanizado por parte de los funcionarios a los usuarios de las IPS evaluadas.

Fuente. Información de las IPS objeto de estudio
Sección 2: El mayor porcentaje de cumplimiento se asocia a la variable servicios generales en un $68 \%$, para profesionales de la salud se observa un incumplimiento del 51\%, lo cual resulta contradictorio ya que los profesionales de la salud son formados para dignificar a los pacientes y brindarles un trato cálido y digno; por último, con un valor porcentual de $42 \%$ se encuentra personal de vigilancia.

De acuerdo al resultado anterior a esta variable corresponde diseñar el mayor número de oportunidades de mejora. (Tabla 2). 


\section{Profesionales de la salud}

" Procurar que los primeros 30 segundos sean claves para empatizar con el paciente ofreciendo un trato atento y cordial.

» Al finalizar la atención, estar prestos a escuchar las inquietudes de los pacientes y allegados, garantizando una escucha activa.

» El respeto de los colaboradores a la privacidad y dignidad, durante el proceso de atención (por ejemplo, mientras se baña y es examinado), para ello la IPS debe provisionar elementos físicos, de infraestructura y de vestuario para que el usuario se sienta cómodo.

» El profesional tiene que explicar en qué consiste el consentimiento informado y garantizar su comprensión por parte del paciente o allegado.

" Contar con horarios de visita diferenciales, de acuerdo a

Personal de vigilancia las necesidades de los usuarios y sus preferencias, es de resaltar que, para mejorar esta variable, se requiere de la decisión de la alta gerencia.

\section{Servicios generales}

» Considerar dietas para los pacientes teniendo en cuenta gustos y preferencias, se hace énfasis en que la mejora de esta variable, depende de la alta gerencia.

Tabla 3. Plan de mejora. Fuente: elaboración propia.

La sección 3, asociada al cumplimiento del trato humanizado en la prestación del servicio presenta los siguientes datos (Gráfica 3).

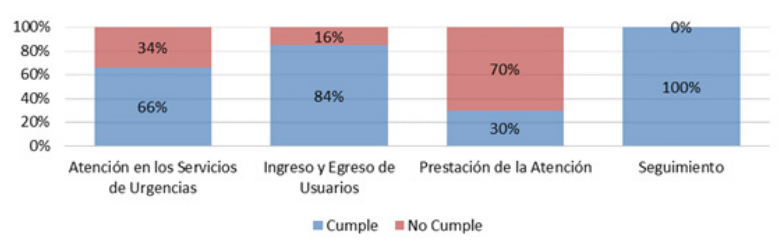

Gráfica 3. Porcentaje de cumplimiento del trato humanizado en la prestación del servicio. Fuente: Información de las IPS objeto de estudio.
Sección 3: Se resalta el valor porcentual de la variable seguimiento en un $100 \%$, a pesar que en esta sección sobresalen valores porcentuales de cumplimiento por encima del 65\% lo que indica un adecuado fortalecimiento para las instituciones de salud, se anota el no cumplimiento de la variable prestación de la atención en un $70 \%$, resultando también contradictorio si se tiene en cuenta que la esencia misional de las IPS, es precisamente la prestación y atención de servicios. En la Tabla 3 se detalla el plan de mejora sugerido para intervenir la sección evaluada. 


\section{Atención en los servicios de urgencias}

»El personal debe saludar al paciente usuario y establecer contacto visual con él.

\begin{abstract}
Ingreso y egreso de usuarios
» Hacer un análisis de las barreras de acceso a la organización.

" Preparar al equipo de salud para realizar al ingreso una identificación de las necesidades específicas de los pacientes y contar con una herramienta para hacerlo.

» Respetar la dignidad al cumplir los protocolos para el ingreso de pacientes que requieran aislamiento.

Prestación de la atención

" Informar a los pacientes sobre sus derechos o a los acompañantes cuando estos no pueden ser entendidos por los pacientes.

» Definir cómo realizar el abordaje respetuoso de las tradiciones, creencias y valores de los usuarios.

» Brindar la información necesaria y suficiente de resultados de los exámenes o los procedimientos diagnósticos y confirmar su entendimiento por parte del usuario y/o su familia, especialmente si se trata de grupos especiales.

» Incluir en el plan de capacitación anual un programa para desarrollar habilidades para la comunicación y el diálogo, incluidas la consideración al transmitir información dolorosa para el paciente y sus familiares.
\end{abstract}

Tabla 4. Plan de mejora. Fuente: elaboración propia.

Continuando con el análisis del trato humanizado en IPS públicas del departamento de Córdoba, se procedió a comparar el cumplimiento de las variables por sección entre las IPS evaluadas, encontrando:

Sección 1: variables según condiciones y situación del paciente (grupos etarios, binomio madre/hijo, discapacitados y enfermos mentales, enfermos terminales, fallecimiento y abandono).

Se tiene que los porcentajes de cumplimiento en las IPS $4(32 \%), 5(20 \%)$ y $8(32 \%)$, generaron mayor número de oportunidades de mejora, sin embargo, las demás IPS obtuvieron una calificación alrededor del 60\% de cumplimiento. Cabe anotar, que para las IPS 2, 4, 7 y 8 no se evaluaron los ítems asociados sobre al trato a pacientes con enfermedades terminales, porque los criterios no aplicaban.

Sección 2: variables relacionadas con los funcionarios (profesionales de los servicios de la salud, personal de vigilancia y servicios generales).

El porcentaje de cumplimiento en las 8 IPS evaluadas fue similar, encontrando que 5 de estas arrojaron valores entre el 58\%, sobresaliendo la IPS 1, con un valor porcentual de cumplimiento del $68 \%$ con respecto a las demás, sin embargo, cabe aclarar que todas las variables generaron oportunidades de mejora.

Sección 3: variables relacionadas con la prestación del servicio (atención en los servicios de urgencias, ingreso y egreso de usuarios, prestación de la atención y seguimiento). 
Existe gran variación con relación al cumplimiento de lo evaluado, encontrando mayor cumplimiento en las IPS 7,2 y 1 con $76 \%, 74 \%$ y $69 \%$ en forma descendente respectivamente, y donde la IPS 5 con $40 \%$ fue la de más bajo rendimiento correspondiendo a esta última mayor cantidad de oportunidades de mejora en lo relacionado con la prestación del servicio.

A continuación, datos comparativos de cumplimiento global del trato humanizado en las IPS evaluadas. (Gráfica 4).

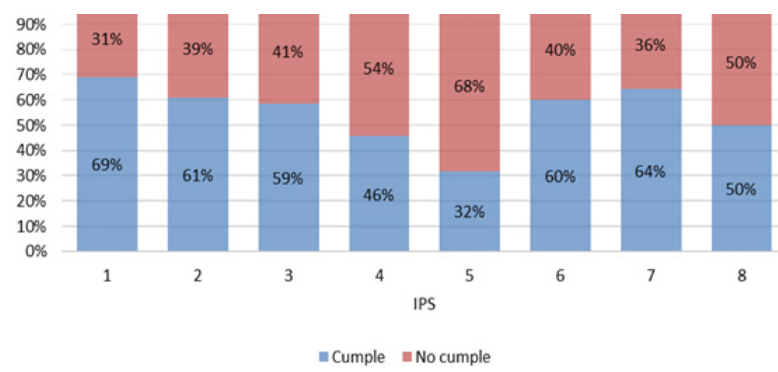

Gráfica 4. Cumplimiento global del trato humanizado desagregado por las IPS evaluadas. Fuente: Información de las IPS objeto de estudio

Los porcentajes indican que las IPS con mayor nivel de cumplimiento en el trato humanizado a los pacientes son las identificadas como 1 y 7 sin embargo, de acuerdo a los resultados todas las instituciones se encuentran por debajo del nivel de cumplimiento deseado que es el $100 \%$, lo anterior se ratifica con el porcentaje global de cumplimiento del trato humanizado en las ocho IPS públicas de un 55\% como mayor valor con una mínima diferencia del 10\% de las IPS que no cumplen, valores que pueden aumentar si se implementan las acciones pertinentes.

De acuerdo al porcentaje de cumplimiento global entre las IPS, la sección con mayores oportunidades de mejora encontrada fue la 1: variables según las condiciones y situación del paciente, con $48 \%$, esto puede deberse a la inexistencia de protocolos, políticas, estrategias, entre otros, que garanticen el trato humanizado a nivel de estas variables.

Mientras, que la sección que presentó menos oportunidades de mejora, fue la 3: variables relacionadas con la prestación del servicio, con un nivel de cumplimiento global del $64 \%$, esto debido a que la mayoría de las IPS tienen fortalecido sus procesos en las unidades funcionales de admisión, urgencias, hospitalización, entre otros, así mismo cuentan con los protocolos necesarios, mecanismos y varias disponen de la asesoría de la oficina de trabajo social, para los casos que aplica. Es importante destacar que dentro de esta sección sobresale en todas las IPS la variable seguimiento por ser la única que garantiza un cumplimiento del $100 \%$ en todas las entidades estudiadas.

\section{DISCUSIÓN}

Este estudio evidencia la necesidad de una atención por parte del profesional de salud generadora de empatía; son los trabajadores en salud los responsables de lograr ese vínculo empático con la persona asistida mediante una comunión intelectual, afectiva y espiritual (13) coincidiendo con Gutiérrez y Triana $(14,15)$ quienes abordan el uso de la empatía para comprender la situación del paciente y así brindarle toda la ayuda posible; y con calidad y equidad como lo concluye Cano. (16)

Para lograr una atención humanizada y con calidad, es imperativo involucrar a todos los colaboradores de una institución en la prestación del servicio lo anterior concordando con lo expresado por González (17) el éxito de las relaciones interpersonales a nivel institucional depende de todos los integrantes del equipo de salud, el cual se concibe integrado por una amplia gama de individuos que va desde el personal de la ambulancia hasta el gerente de la institución". Y a lo que Yepes (18) llama "encuentros dispares". 
Los resultados obtenidos, con relación a la atención por parte del equipo profesional, se consideran contradictorios; teniendo en cuenta que los profesionales de salud de IPS, tanto asistenciales, como administrativos, deben comprender que la atención humanizada es el eje transversal en la prestación de servicios para lograr el mejoramiento continuo en la atención de los pacientes, sus familias y la imagen de las instituciones como lo expone Rodríguez (19). Lo anterior apoyado por la normatividad vigente en salud, la cual está encaminada a mejorar la prestación de los servicios de salud, desde un abordaje integral del paciente-usuario-persona con estándares de calidad; a la cual debe adicionarse en la búsqueda del mejoramiento continuo un trato amable y cálido Concha (20).

\section{CONCLUSIÓN}

Esta investigación determinó evalúo la atención humanizada en IPS públicas de cinco municipios del departamento de Córdoba durante el año 2016, sus resultados permitieron el reconocimiento de oportunidades de mejora, las cuales al ser intervenidas lograrían el cumplimiento ideal de la prestación de una atención en salud humanizado en las instituciones objetos de estudio.

Con el propósito de brindar un servicio de calidad con sentido humano y mayor calidez, se requiere un gran compromiso de la alta gerencia en la promoción de estrategias que garanticen el fomento de la cultura del trato humanizado en la organización, compromiso que debe verse reflejado en la implementación de planes de mejora con la asignación de recursos financieros en lo relacionado con infraestructura e inclusión de elementos de humanización en el ambiente físico en el que se atiente al paciente.

Además, desde la dependencia de talento humano es necesario un proceso de selección de personal teniendo en cuenta habilidades de comunicación efectiva, trabajo en equipo y em- patía, para garantizar colaboradores comprometidos en una atención más humana dentro de la institución.

\section{RECOMENDACIONES}

Se recomienda ampliar el presente estudio en otros municipios, así como también incluir IPS de carácter privado.

\section{CONFLICTO DE INTERÉS}

Las autoras declaran no tener conflictos de interés.

\section{AGRADECIMIENTOS}

A las IPS objeto de estudio por su colaboración en el desarrollo de este trabajo. A la Universidad de Córdoba, por su apoyo en la ejecución de este proyecto de investigación, producto de convocatoria interna.

\section{REFERENCIAS}

1. Barrientos GJ, Marín CA, Becerra RL, Tobón AM. La evaluación de nuevas tecnologías en salud en hospitales: revisión narrativa. Medicina U.P.B. [internet]. 2016 [Consultado 10 de junio de 2019]; (35) Disponible en:http://www.redalyc.org/articulo.oa

2. García CM. Humanizar la asistencia en los grandes hospitales: un reto para el profesional sanitario. Metas Enferm 2014; 17(1): 70-74

3. Correa ZM. La humanización de la atención en los servicios de salud: un asunto de cuidado. Revista Cuidarte. 2016; 7(1), 1210-1218.

4. Rodríguez MJ, Chamorro FH. Diseño de un programa de atención humanizada para la prestación del servicio de salud en urgencias pediátricas en una IPS de Cali. Ciencia \& Salud. 2015; 3(12):45-52 
5. Observatorio de calidad de la atención en salud, 2016. Recuperado de https://www. minsalud.gov.co/sites/rid/Lists/BibliotecaDigital/RIDE/DE/CA/Guia-metodologica-observatorio-calidad.pdf

6. Resolución 1445 de 2006 -Ministerio de la Protección Social. (2006, mayo 8). Diario Oficial No. 46.271. Bogotá, Colombia: Imprenta Nacional.

7. Ministerio de salud y protección social. (3 de abril del 2006). Por el cual se establece el Sistema Obligatorio de Garantía de Calidad de la Atención de Salud del Sistema General de Seguridad Social en Salud. Decreto 1011 de 2006. Recuperado de https://www.minsalud.gov.co/Normatividad_ Nuevo/DECRETO\%201011\%20DE\%20 2006.pdf

8. Ministerio de Salud y Protección Social República de Colombia. (28 de mayo del 2014). Artículo 6. (Requisitos para la inscripción y habilitación de servicios de salud en el REPS.) resolución 2003 del 2014. Recuperado de https://www.minsalud.gov. co/sites/rid/Lists/BibliotecaDigital/RIDE/DE/ DIJ/Resolucion-2003-de-2014.pdf

9. Ministerio de Salud y Protección Social. Resolución 123 de 2012. Por la cual se modifica el artículo 2 de la resolución 1445 de 2008

10. Programa de Auditoría para el mejoramiento de la Calidad PAMEC.

11. Mojica, G, W. La Gerencia Social en La Humanización a la Atención en Salud, Bogotá: Escuela Superior de Administración Pública, 2007.
12. Oger SN. La humanización de la salud y las nuevas tecnologías. Revista Iberoamericana de Bioética. 2018; (8), 01-11.

13. Gutiérrez FR. La humanización de (en) la Atención Primaria Revista Clínica de Medicina de Familia. Sociedad Castellano-Manchega de Medicina de Familia y Comunitaria Albacete, España. 2017; (10) 29-38

14. Triana Restrepo, M. C. (2017). La empatía en la relación enfermera-paciente. Avances en Enfermería, 35(2), 121-122.

15. Cano SM, Giraldo A, Forero C. Concepto de calidad en salud: resultado de las experiencias de la atención, Medellín, Colombia. Rev. Fac. Nac. Pública 2016; 34(1): 4853. DOI: 10.17533/udea.rfnsp.v34n1a06

16. González M. Hospital Psiquiátrico de la Habana la relación equipo de salud-paciente-familiar Revista Cubana Salud Pública v.32 n.3 Ciudad de La Habana jul.-sep. 2006

17. Yepes CE, Giraldo AJ, Botero N., Guevara JC. En búsqueda de la atención: necesidades en salud, itinerarios y experiencias. Hacia Promoc. Salud. 2018; 23 (1): 88-105. DOI: 10.17151/hpsal.2018.23.1.7

18. Rodríguez MJ, Chamorro FH. Diseño de un programa de atención humanizada para la prestación del servicio de salud en urgencias pediátricas en una IPS de Cali. Ciencia \& Salud. 2015; 3(12):45-52

19. Concha MX, Urrutia MT. Calidad de atención Programa AUGE cáncer cérvicouterino: diferencias y similitudes entre usuarias y profesionales. Rev.Chil. Obs.Ginecol., 2011; 76(5):294-301. 